\title{
Avaliação da independência funcional e da qualidade de vida no pós-operatório de tumor cerebral
}

\author{
Assessment of functional independence and quality of life after surgery for brain tumor
}

\begin{abstract}
Juliana Noronha de Pieri ${ }^{1}$, Caroline Háruka Giriko ${ }^{1}$, Márcia Helena dos Santos Abranches', Heloise Cazangi Borges ${ }^{3}$, Therezinha Rosane Chamlian ${ }^{4}$
\end{abstract}

\section{RESUMO}

Objetivo. Verificar os efeitos da fisioterapia na independência funcional e na qualidade de vida em pacientes no pós-operatório de tumor cerebral. Método. Participaram deste estudo 6 indivíduos no pós-operatório de tumor cerebral internados na enfermaria de neurocirurgia do Hospital São Paulo (HSP) no período de julho a dezembro de 2009. Foram mensuradas a independência funcional e a qualidade de vida por meio da Medida de Independência Funcional (MIF) e da Versão Brasileira do questionário de qualidade de vida (SF-36), respectivamente, logo após a cirurgia e ao término das intervenções fisioterapêuticas, realizadas por 5 dias consecutivos durante $45 \mathrm{minu}$ tos. Resultados. Os indivíduos apresentaram uma média de idade de $65 \pm 10.43$ anos e $50 \%$ dos pacientes $(n=3)$ apresentaram comprometimento motor de hemiparesia à esquerda. Ao término das intervençóes fisioterapêuticas, observou-se na MIF um aumento de sua pontuaçáo em $66.68 \%$ dos pacientes $(n=4)$ variando entre 7 e 34 pontos e no SF-36 uma manutençáo de sua pontuação em cerca de $50 \%(n=3)$ a $83.35 \%(n=5)$ dos pacientes. Conclusáo. A maioria dos pacientes em pós-operatório de tumor cerebral apresentou um aumento de sua independência funcional, bem como, uma manutenção de sua qualidade de vida ao término das intervençóes fisioterapêuticas.

Unitermos. Neoplasias Encefálicas, Avaliação da Deficiência, Qualidade de Vida, Fisioterapia, Reabilitação.

Citaçáo. Pieri JN, Abranches MHS, Giriko CH, Borges HC, Chamlian TR. Avaliação da independência funcional e da qualidade de vida no pós-operatório de tumor cerebral.

\begin{abstract}
Objective. The objective of this study was to verify the effects of physiotherapy on functional independence and quality of life in patients after surgery for brain tumor. Method. 6 subjects participated in this study in postoperative brain tumor admitted to the neurosurgery nurse of the Hospital São Paulo (HSP) in the period from July to December 2009. We measured the functional independence and quality of life through the Functional Independence Measure (FIM) and the Brazilian Version of the quality of life questionnaire (SF-36), respectively, immediately after surgery and at the end of physical therapy interventions, carried out for 5 consecutive days for 45 minutes. Results. Subjects had a mean age of $65 \pm 10.43$ years and $50 \%$ of patients $(n=3)$ showed motor impairment of left hemiparesis. At the end of physical therapy interventions was observed in MIF increased its score in $66.68 \%$ of patients $(n=4)$ ranging from 7 to 34 points and the SF-36 maintained its score by $50 \%(n=3)$ to $83.35 \%(n=5)$ patients. Conclusion. Most patients in postoperative brain tumor showed an increase in its operational independence, as well, maintaining their quality of life at the end of physical therapy interventions.
\end{abstract}

Keywords. Brain Neoplasms, Disability Evaluation, Quality of Life, Physical Therapy, Rehabilitation.

Citation. Pieri JN, Abranches MHS, Giriko CH, Borges HC, Chamlian TR. Assessment of functional independence and quality of life after surgery for brain tumor.

\footnotetext{
Trabalho realizado no Hospital Sáo Paulo (HSP), Sáo Paulo-SP, Brasil. 1. Fisioterapeuta Especializada em Fisioterapia Motora Hospitalar e Ambulatorial aplicada à Neurologia da Universidade Federal de São Paulo (UNIFESP), São Paulo-SP, Brasil.

2. Fisioterapeuta Especializada em Fisioterapia Motora Hospitalar e Ambulatorial aplicada à Neurologia, Fisioterapeuta do Hospital São Paulo e Preceptora do Curso de Especialização em Fisioterapia Motora Hospitalar e Ambulatorial aplicada à Neurologia da UNIFESP, São Paulo-SP, Brasil.

3. Fisioterapeuta do Lar Escola Sáo Francisco (LESF), Coordenadora e Preceptora do Curso de Especializaçáo em Fisioterapia Motora Hospitalar e Ambulatorial aplicada à Neurologia da UNIFESP, São Paulo-SP, Brasil.

4. Fisiatra, Doutora, Professora Afiliada, Chefe de Clínica da Disciplina de Fisiatria do Departamento de Ortopedia e Traumatologia da UNIFESP e Diretora Técnica do Lar Escola São Francisco (LESF), São Paulo-SP, Brasil.
}

Endereço para correspondência: Juliana N Pieri Rua Jaci, 118/32 CEP 04140-080, São Paulo-SP, Brasil. E-mail: juli3np@hotmail.com

Relato de Caso Recebido em: 07/05/10 Aceito em: 10/08/10 Conflito de interesses: não 


\section{INTRODUÇÃO}

A incidência de tumores cerebrais, primários e metastáticos, vem crescendo com bastante frequência e, consequentemente, o número de pacientes que necessitam de serviços de reabilitação ${ }^{1}$.

Nos Estados Unidos, no ano de 2006, foi estimado um número de 18.800 novos casos de tumor cerebral, além de 12.800 óbitos ocasionados pelo tumor ${ }^{2}$. No Brasil, em um trabalho realizado no Departamento de Patologia da Universidade Federal de São Paulo (UNIFESP), em um período de 15 anos, foi encontrada uma incidência de 1,14\% de neoplasias em material de biópsias, sendo 90,3\% de neoplasias primárias e 9,7\% de metastáticas. As neoplasias primárias mais frequentes foram: os meningiomas, schwannomas e o astrocitomas de baixo grau. Cerca de $70 \%$ das neoplasias intracranianas primárias em adultos são supratentoriais ${ }^{3}$.

Os tumores cerebrais originam inúmeros comprometimentos neurológicos que variam em relação ao seu tamanho, sítio da lesão e a qualidade invasiva do tumor. Além disso, os déficits funcionais desses pacientes não são ocasionados somente pelo tumor em si, mas também pelas sequelas do tratamento com a radio e a quimioterapia ${ }^{2,4}$.

As metástases extracranianas de neoplasias primárias do sistema nervoso central são raras; Sendo justificadas devido à ausência de vasos linfáticos; a resistência dos seios venosos durais à invasão tumoral; o colapso das veias cerebrais prévio à invasão de células tumorais; a incapacidade de proliferação de células gliais noutros tecidos e a curta sobrevida destes pacientes 5 . Porém, quando estas ocorrem, sua disseminação é feita no próprio parênquima cerebral e por disseminação pela via liquórica ${ }^{2,3}$.

O fator prognóstico de maior importância dos tumores cerebrais é a localização, entretanto, este também se baseia em fatores genéticos, distribuição histológica e nos tipos de tratamentos realizados ${ }^{6}$.

Recentemente, devido aos avanços das técnicas de quimioterapia e radioterapia, por meio da implantação de "wafers" quimioterápicos, "gamma Knife”, radiocirurgia esterotáxica e a braquiterapia, vem ocorrendo um aumento em até 15 vezes da sobrevida de pacientes com tumor. No entanto, esta sobrevida é acompanhada por sequelas neurológicas que aumentam o número de pacientes que necessitam de serviços de reabilitação ${ }^{4,6,7}$.
Essas técnicas também intervêm significativamente no programa de reabilitação e na qualidade de vida desses indivíduos, uma vez que a fadiga é um sintoma comumente encontrado após a radioterapia, e que a ansiedade e a depressão são frequentemente encontradas nos pacientes que realizam quimioterapia ${ }^{8-10}$.

Mas, apesar da interferência destes fatores, os comprometimentos vistos em indivíduos com tumor cerebral são comumente observados em pacientes com outras doenças neurológicas ${ }^{1}$. Visto que, tanto em tumores primários como metastáticos, podem ser encontrados em $80 \%$ dos pacientes comprometimentos cognitivos, $78 \%$ fraqueza muscular, 53\% déficits visuais e perceptuais, $38 \%$ perda sensorial e em $37 \%$ disfunção no intestino e na bexiga. Outros déficits neurológicos encontrados com menor frequência são: a paralisia dos nervos cranianos, ataxia, afasia, disfagia, disartria e a diplopia ${ }^{10,11}$.

Adjunto aos comprometimentos funcionais, os pacientes com tumor cerebral ainda apresentam consideráveis afliçôes, particularmente entre o início e os estágios finais da doença, uma vez que estes vivenciam confrontos com a própria doença, adaptaçôes devido às limitaçôes funcionais e sociais e às decisóes dos múltiplos tratamentos ${ }^{9,12}$.

Atualmente, algumas pesquisas sugerem que os pacientes com diferentes tipos de tumores cerebrais alcançam bons ganhos funcionais após a reabilitação $0^{2,4,10,13,14}$.

Muito tem sido descrito sobre a sobrevivência após um tumor cerebral, mas, poucos são os relatos sobre o desempenho funcional, a qualidade de vida e os seus resultados obtidos após um programa de reabilitação.

Deste modo, o presente estudo, tem o objetivo de verificar os efeitos da fisioterapia na independência funcional e na qualidade de vida em pacientes no pós-operatório de tumor cerebral, internados na enfermaria neurocirúrgica do HSP.

\section{MÉTODO}

Trata-se de um estudo de relatos de casos, realizado na enfermaria de neurocirurgia do HSP. O estudo obteve aprovação do Comitê de Ética em Pesquisa da UNIFESP (Processo $n^{\circ}$ 0856/2009).

\section{Sujeitos}

Participaram deste estudo 6 indivíduos no pós- 
operatório de tumor cerebral, internados na enfermaria de neurocirurgia do HSP.

Foram considerados critérios de inclusão: indivíduos com idade acima de 18 anos; diagnóstico clínico e radiológico de tumor cerebral; submetidos à intervenção neurocirúrgica no período de julho a dezembro de 2009; presença de déficits motores no pós-operatório de neurocirurgia (pontuação igual ou menor a 4 de acordo com a Escala de Kendall ${ }^{15}$ ); concordância e assinatura do Termo de Consentimento Livre e Esclarecido dos sujeitos envolvidos na pesquisa.

Foram considerados critérios de exclusão: pacientes politraumatizados ou com complicações associadas, tais como: rebaixamento do nível de consciência, aumento da pressão intracraniana, instabilidade hemodinâmica; presença de afasia de expressão e/ou compreensão e alteraçōes cognitivas que interferissem no entendimento da intervenção fisioterapêutica.

\section{Procedimento}

No período pré e pós-intervenção fisioterapêutica foram mensuradas a independência funcional e a qualidade de vida, respectivamente, por meio da Medida de Independência Funcional (MIF) e da Versão Brasileira do Questionário de Qualidade de Vida (SF-36).

A MIF é um instrumento de avaliação da incapacidade de pacientes com restriçôes funcionais de origem variada. Seu objetivo é avaliar de maneira quantitativa o desempenho funcional do indivíduo para a realização de um conjunto de 18 tarefas. Entre as tarefas avaliadas estão os autocuidados, transferências, locomoção, controle esfincteriano, comunicação e cognição social, que inclui memória, interação social e resolução de problemas. Cada uma dessas atividades é pontuada em uma escala de graus de dependência de 7 níveis, em que 1 corresponde à dependência total e 7 corresponde à independência completa, assim a pontuação total varia de 18 a 126 pontos $^{16}$.

O SF-36 é um instrumento genérico de avaliação da qualidade de vida. Trata-se de um questionário multidimensional formado por 36 itens englobado em 8 escalas ou componentes sendo estes: capacidade funcional, aspectos físicos, dor, estado geral da saúde, vitalidade, aspectos sociais, aspectos emocionais e saúde mental. Apresenta um escore final de 0 a 100, no qual 0 corresponde a pior estado geral de saúde e 100 a melhor estado de saúde ${ }^{17}$.

A primeira aplicação de ambas as escalas foi realizada no pós-operatório prontamente após o retorno do paciente da Unidade de Terapia Intensiva (UTI) à enfermaria de neurocirurgia, independente do dia de pós-operatório.

Posteriormente foi realizada em todos os pacientes, uma intervenção fisioterapêutica a qual consistiu em cinco sessôes com duração de quarenta e cinco minutos, uma vez por dia durante cinco dias consecutivos. Tal intervenção, realizada individualmente nos pacientes, foi composta por:

1. Exercícios para o ganho de flexibilidade muscular por meio de alongamentos musculares de membros superiores, tronco e membros inferiores durante 6 minutos, com 1 série de 30 segundos de permanência em cada alongamento (apenas no hemicorpo comprometido) ${ }^{1,18}$.

2. Exercícios terapêuticos podendo ser passivo, ativo-assistido, ativo-livre ou resistido, no hemicorpo comprometido, dependendo das condiçóes motoras do paciente durante 6 minutos com 2 séries de 10 repetiçôes ${ }^{1,18,19}$.

3. Exercícios para o ganho de controle de tronco em sedestação durante 7 minutos, visando à manutenção da postura e a mobilidade na mesma durante o apontar ou alcançar objetos nos três planos de movimento (sagital, frontal e transverso) $)^{1,18,19}$.

4. Treino das atividades motoras funcionais como o rolar e as transferências de decúbito dorsal para sedestação e de sedestação para o ortostatismo, no leito por 8 minutos $^{1,18,19}$. 5. Exercícios para o treino de equilíbrio durante 6 minutos, os quais constavam em: pegar objetos em diferentes posiçôes, pegar objetos no chão, andar entre linhas e a manutençâo da postura durante a mudança da base de suporte ao sentar e levantar ou ao manter um pé na frente do outro ${ }^{20}$.

6. Treino de marcha, realizado nos pacientes que apresentaram potencial para a deambulação, associado ao uso de auxiliares sendo estes: andador ou muleta ou bengala por 12 minutos $^{1,18,19}$.

7. Posicionamento correto do paciente no leito por meio de coxins e travesseiros ${ }^{1,18,19}$.

No último dia da intervenção fisioterapêutica, ou seja, no quinto dia, logo após o término da intervenção, as escalas foram reaplicadas. 
As atividades do presente protocolo foram selecionadas atendendo às necessidades individuais de cada paciente de acordo com o seu respectivo quadro motor e nível de independência funcional para realização dos exercícios.

\section{RESULTADOS}

Com relação às características da amostra de acordo com o sexo, idade e dominância, ressaltou-se a predominância do sexo masculino e a dominância no lado direito em $100 \%$ da amostra $(n=6)$, além disso, os indivíduos apresentaram uma idade mínima de 44 anos e máxima de 72 anos, assim, a média de idade encontrada foi de $65 \pm 10.43$ anos.

Já nas características da amostra de acordo com o tipo de tumor, localização, dia de pós-operatório na admissão da intervenção fisioterapêutica e comprometimento motor, foi possível observar que a média do dia de pós-operatório em que os pacientes iniciaram o tratamento fisioterapêutico foi de $6.34 \pm 1.21$ dias. Tais dados estão explanados no Quadro 1.

Nos resultados da MIF, verificou-se que $100 \%$ dos pacientes $(n=6)$ apresentaram pontuação máxima do domínio MIF cognitivo, tanto na avaliação pré como pósintervenção fisioterapêutica.
De acordo com a variação da pontuação total da MIF entre as avaliaçôes pré e pós-intervenção fisioterapêutica, notou-se que $66.68 \%$ dos pacientes $(n=4)$, obtiveram um aumento na pontuação ao término da intervenção fisioterapêutica, variando entre 7 e 34 pontos, no entanto, $33.34 \%$ dos pacientes $(\mathrm{n}=2)$ mantiveram sua pontuação inalterada ao término da intervenção. Esses dados estão ilustrados na Tabela 1.

Nas mensuraçóes do SF-36, os domínios limitação por aspectos físicos e emocionais apresentaram-se zerados em $100 \%$ dos pacientes ( $n=6)$, tanto na avaliação pré como pós-intervenção fisioterapêutica, por conseguinte, não foi possível realizar uma variação da pontuação para estes domínios.

E, referente aos demais domínios: capacidade funcional, dor, estado geral de saúde, vitalidade, aspectos sociais e saúde mental, notou-se que cerca de $50 \%(\mathrm{n}=3)$ a 83.35\% ( $\mathrm{n}=5)$ dos pacientes mantiveram suas pontuaçóes inalteradas nos diferentes períodos de avaliações (pré e pós).

No entanto, $33.34 \%$ da amostra $(n=2)$ demonstraram uma diminuição de 7 pontos do domínio capacidade funcional na avaliação pós-intervenção fisioterapêutica; Já o domínio estado geral, $16.67 \%$ da amostra $(\mathrm{n}=1)$, apresentou-se com uma diminuição de 8 pontos na avaliação pós-intervenção fisioterapêutica.

Quadro 1

Características da amostra de acordo com o tipo de tumor, localização, dia de pós-operatório na admissão da intervenção fisioterapêutica e comprometimento motor

\begin{tabular}{|c|c|c|c|c|}
\hline Pacientes & Tipo de Tumor & Localizaçáo & $\begin{array}{c}\text { Dia de } \\
\text { Pós-operatório }\end{array}$ & Comprometimento Motor \\
\hline Paciente 1 & Schwannoma & $\begin{array}{l}\text { Ângulo } \\
\text { cerebelopontino }\end{array}$ & $\begin{array}{l}8^{\circ} \mathrm{PO} \text { de ressecção } \\
\text { parcial do tumor }\end{array}$ & $\begin{array}{l}\text { Ataxia e paralisia do V e VII } \\
\text { nervo craniano }\end{array}$ \\
\hline Paciente 2 & $\begin{array}{l}\text { Oligodendroma } \\
\text { Anaplásico } \\
\text { (recidiva) }\end{array}$ & Lobo temporal à D & $\begin{array}{l}5^{\circ} \mathrm{PO} \text { de ressecçáo } \\
\text { total do tumor }\end{array}$ & $\begin{array}{l}\text { Hemiparesia incompleta } \\
\text { desproporcionada de predomínio } \\
\text { braquial à } \mathrm{E}\end{array}$ \\
\hline Paciente 3 & $\begin{array}{l}\text { Glioblastoma } \\
\text { Multiforme }\end{array}$ & $\begin{array}{l}\text { Lobo frontal e pari- } \\
\text { etal à D }\end{array}$ & $\begin{array}{l}7^{\circ} \mathrm{PO} \text { de ressecçáo } \\
\text { total do tumor }\end{array}$ & $\begin{array}{l}\text { Hemiparesia incompleta } \\
\text { desproporcionada de predomínio } \\
\text { braquial à } \mathrm{E}\end{array}$ \\
\hline Paciente 4 & $\begin{array}{l}\text { Glioblastoma } \\
\text { Multiforme }\end{array}$ & Lobo frontal à $\mathrm{D}$ & $\begin{array}{l}\text { 7o PO de ressecçáo } \\
\text { total do tumor }\end{array}$ & $\begin{array}{l}\text { Hemiparesia incompleta } \\
\text { desproporcionada de predomínio } \\
\text { braquial à } \mathrm{E}\end{array}$ \\
\hline Paciente 5 & $\begin{array}{l}\text { Meningioma } \\
\text { (recidiva) }\end{array}$ & Lobo parietal à $\mathrm{E}$ & $\begin{array}{l}\text { 6o PO de ressecçáo } \\
\text { total do tumor }\end{array}$ & $\begin{array}{l}\text { Hemiparesia incompleta } \\
\text { desproporcionada de predomínio } \\
\text { braquial à D }\end{array}$ \\
\hline Paciente 6 & $\begin{array}{l}\text { Glioblastoma } \\
\text { Multiforme }\end{array}$ & $\begin{array}{l}\text { Lobo frontal e pari- } \\
\text { etal à E }\end{array}$ & $\begin{array}{l}5^{\circ} \mathrm{PO} \text { de ressecçáo } \\
\text { total do tumor }\end{array}$ & $\begin{array}{l}\text { Hemiparesia incompleta } \\
\text { desproporcionada de predomínio } \\
\text { braquial à D }\end{array}$ \\
\hline
\end{tabular}

E: esquerda, D: direita, PO: pós-operatório 
Tabela 1

Variação da pontuação da MIF entre a avaliação pré e pós-intervenção fisioterapêutica

\begin{tabular}{cccc}
\hline Pacientes & $\begin{array}{c}\text { Avaliaçáo } \\
\text { Pré-intervenção }\end{array}$ & $\begin{array}{c}\text { Avaliação } \\
\text { Pós-intervenção }\end{array}$ & $\begin{array}{c}\text { Variação da } \\
\text { pontuaçáo da } \\
\text { escala MIF entre a } \\
\text { avaliação pré e pós- } \\
\text { intervençáo }\end{array}$ \\
\hline Paciente 1 & 73 & 107 & 34 \\
Paciente 2 & 100 & 100 & 0 \\
Paciente 3 & 67 & 74 & 7 \\
Paciente 4 & 100 & 100 & 0 \\
Paciente 5 & 91 & 103 & 12 \\
Paciente 6 & 68 & 95 & 27 \\
\hline
\end{tabular}

\section{DISCUSSÃO}

Os dados deste estudo condizem com os achados da literatura, que relatam um acometimento maior do tumor cerebral entre a quinta e a sétima década de vida, além de uma maior incidência na população masculina, em uma proporção de 2:1 em relação às mulheres na maioria dos tumores ${ }^{7,21}$.

Estudos indicam que o glioblastoma multiforme é o tipo de tumor primário mais comumente encontrado representando cerca de $50 \%$ de todos os gliomas ${ }^{3,6}$. Os meningiomas também se apresentam como um tipo de tumor primário frequente, o qual compreende cerda de $27,4 \%$ de todos os tumores. Já o oligodendroma representa $23 \%$ e o schawannoma abrange apenas $10 \%$ de todos os tumores intracranianos ${ }^{22}$.

A literatura comprova que o glioblastoma multiforme, assim como o oligodendroglioma, são frequentemente localizados no lobo frontal, porém, podem ocorrer no lobo temporal, parietal e occipital, além disso, o schawannoma é normalmente localizado no canal auditivo interno, mas também pode se estender para o ângulo cerebelopontino $^{7,21}$.

Alguns pesquisadores ${ }^{4}$ averiguaram a incidência dos déficits neurológicos em pacientes com tumor cerebral e destacaram a hemiparesia e a tetraparesia como um dos déficits mais comumente encontrados e a ataxia e a paralisia do V e VII nervo craniano como os déficits motores de menor ocorrência.

Assim como no presente estudo, achados clínicos evidenciam que pacientes com tumor cerebral obtêm um aumento na independência funcional após um programa de reabilitaçãa ${ }^{2,4,10,13,14}$.

A melhora funcional observada por meio da MIF, corrobora com alguns autores ${ }^{6}$ que verificaram após um programa de reabilitação uma melhora significativa nos resultados funcionais do MIF total, motor e cognitivo nos pacientes com tumores cerebrais primários e metastáticos. O mesmo estudo concluiu que o aumento significativo na MIF é um fator preditivo para melhora na sobrevida em pacientes com glioblastoma multiforme e tumores metastáticos.

A partir dessa informação ressaltam-se cada vez mais a importância da realização de um tratamento fisioterapêutico no período hospitalar para que, dessa forma, os pacientes com diferentes tipos de tumor cerebral possam se beneficiar de uma sobrevida com aumento de sua independência funcional o mais breve possível.

Atualmente estudos comprovam que fatores como: tipos de tumor, local da lesão e realização de tratamentos concomitantes com quimio ou radioterapia, não influenciam significantemente nos resultados funcionais desses indivíduos $^{2,6}$. Essas informaçóes vão de encontro com o presente estudo, o qual encontrou ganhos funcionais em tumor de diferentes tipos e localizaçóes.

Em contrapartida, outro estudo ${ }^{1}$ demonstra que pacientes que realizam tratamento de radioterapia concomitante com a fisioterapia, obtém um melhor ganho funcional do que os que não fazem radiação e que pacientes com tumores cerebrais recorrentes apresentam ganhos funcionais significativamente menores do que os pacientes com uma única ocorrência do tumor.

Valendo-se de um protocolo semelhante ao deste estudo, pesquisadores ${ }^{2}$ encontraram após o programa de reabilitação uma melhora significativa na capacidade funcional e locomotora dos pacientes com tumor cerebral e AVC, porém o grau de melhora não diferiu entre os dois grupos sugerindo que pacientes com tumor cerebral apresentam ganhos funcionais e comprometimentos motores semelhantes aos dos pacientes com AVC.

Contudo, vale lembrar que mesmo com a presença e semelhança desses déficits, o papel da fisioterapia no pósoperatório de tumor cerebral, não se apresenta totalmente estabelecido como em outras doenças neurológicas ${ }^{2,10}$.

Referente ao domínio MIF cognitivo, sua pontuação máxima observada em $100 \%$ da amostra $(n=6)$ pode 
ser justificada devido aos critérios de exclusão deste estudo, que excluíam pacientes com afasia de compreensão e/ou expressão e distúrbios cognitivos que impedissem o entendimento da intervenção, logo, era prevista que a pontuação no período pré-intervenção fosse inalterada ao término do programa fisioterapêutico.

Já os resultados obtidos com relação ao SF-36, confirmam o de estudo anterior ${ }^{9}$, que apesar de encontrarem um aumento significativo da MIF ao término do programa de reabilitação, não puderam observar, por meio da "Functional Assessment of Cancer Therapy-Brain" (FACT-BR), uma melhora na qualidade de vida desses indivíduos durante o período da reabilitação, porém, após o segmento de 3 meses os autores notaram uma melhora significativa na qualidade de vida desses pacientes.

Os domínios: limitação por aspecto físico e aspecto emocional apresentaram-se zerados em ambas as avaliaçóes. Esses resultados vão de encontro com estudo prévio $^{22}$ o qual demonstra que problemas de natureza emocional, como depressão, raiva e fadiga podem ocasionar um impacto negativo tanto nos aspectos físicos como psicológicos de pacientes com tumor cerebral, interferindo, deste modo, na participação desses indivíduos no trabalho e em outras atividades de vida diária.

Estudos destacam que a qualidade de vida tende a ser maior em pacientes com tumores benignos, enquanto que pacientes com tumores malignos tendem a apresentar uma piora em sua qualidade de vida. Além disso, o grau de envolvimento do paciente com a doença, bem como, os tipos de tratamentos realizados podem ter impactos negativos na qualidade de vida, uma vez que a fadiga pode piorar com a radioterapia (como observado em pacientes com gliomas malignos) e, a ansiedade e a depressão podem aumentar nos pacientes que recebem quimioterapia $^{8,12,22}$.

Apesar dos pacientes deste estudo, não terem realizado tratamento complementar com quimio ou radioterapia, sabe-se, no entanto, que a deficiência funcional decorrente do tumor cerebral por si só acarreta em uma considerável angústia, sobretudo nos estágios iniciais e finais da doença e, ainda somados a este fator, os pacientes apresentam uma redução em sua qualidade de vida devido a conflitos perante a própria doença, adaptaçôes às limitações funcionais e às decisôes devido aos múltiplos tratamentos realizados ${ }^{22}$.

\section{CONCLUSÃO}

A maioria dos pacientes em pós-operatório de tumor cerebral apresentou um aumento de sua independência funcional, bem como, uma manutenção de sua qualidade de vida ao término das intervençôes fisioterapêuticas.

No entanto, convém ressaltar a necessidade de um maior período de tratamento fisioterapêutico e de um segmento para verificar se os resultados dos ganhos funcionais e da qualidade de vida serão mantidos ou acrescentados após a concretização do programa fisioterapêutico.

E, devido à pequena amostra deste estudo $(n=6)$, salienta-se a necessidade de um estudo com um número maior de sujeitos para constatar, por meio de uma análise estatística, resultados mais significativos com relação à efetividade da fisioterapia em pacientes em pós-operatório de tumor cerebral.

\section{REFERÊNCIAS}

1.Marciniak CM, Sliwa JA, Heinemann AW, Semik PE. Functional outcomes of persons with brain tumors after inpatient rehabilitation. Arch Phys Med Rehabil 2001;82:457-63.

http://dx.doi.org/10.1053/apmr.2001.21862

2.Geler-Kulcu D, Gulsen E, Buyukbaba E, Ozkan D. Functional recovery of patients with brain tumor or acute stroke after rehabilitation: A comparative study. J Clin Neurosci 2009;16:74-8.

http://dx.doi.org/10.1016/j.jocn.2008.04.014

3.Stávale JN. Gliomas cerebrais. In: Braga FM, Melo PMP. Guias de medicina ambulatorial e hospitalar UNIFESP/ Escola Paulista de Medicina: neurocirurgia. São Paulo: Editora Manole; 2005, p.87-94.

4.Mukand JA, Blackinton DD, Crincoli MG, Lee JJ, Santos BB. Incidence of neurologic deficits and rehabilitation of patients with brain tumors. Am J Phys Med Rehabil 2001;80:346-50.

http://dx.doi.org/10.1097/00002060-200105000-00005

5.Santos AV, Saraiva PF, Santiago B. Metastização extracraniana de Glioblastoma Multiforme. Acta Med Port, 2003;16:209-11.

6.Tang V, Rathbone M, Dorsay JP, Jiang S, Harvey D. Rehabilitation in primary and metastatic brain tumours: Impact of functional outcomes on survival. J Neurol 2008;255:820-7.

http://dx.doi.org/10.1007/s00415-008-0695-z

7.Christiansen CJ, Lopez RO, Phillips KM. Tumores cerebrais. In: Umphred, DA. Reabilitação neurológica. 4a . ed. São Paulo: Editora Manole, 2004, p.734-53. 8.Kirshblum S, O’Del WM, Ho C, Barr K. Rehabilitation of persons with central nervous system tumors. Câncer 2001;92:1029-38.

http://dx.doi.org/10.1002/1097-0142(20010815)92:4+<1029::AIDCNCR1416>3.0.CO;2-P

9.Huang ME, Wartella JE, Kreutzer JS. Functional outcomes and quality of live in patients with brain tumors: a preliminary report. Arch Phys Med Reha- 
bil 2001;82:1540-5.

http://dx.doi.org/10.1053/apmr.2001.26613

10.Greenberg E, Treger I, Ring H. Rehabilitation outcomes in patients with brain tumors and acute stroke: comparative study of inpatient rehabilitation. Am J Phys Med Rehabil 2006;85:568-73.

http://dx.doi.org/10.1097/01.phm.0000223218.38152.53

11.Giordana MT, Clara E. Functional rehabilitation and brain tumour patients. A review of outcome. Neurol Sci 2006;27:240-4.

http://dx.doi.org/10.1007/s10072-006-0677-9

12. Heimans JJ, Taphoorn MJB. Impact of brain tumour treatment on quality of life. J Neurol 2002;249:955-60.

http://dx.doi.org/10.1007/s00415-002-0839-5

13.Grisold W, Oberndorfer S, Hitzenberger P. Brain tumour treatment: the concept of inter- and multidisciplinary treatment. Wien Med Wochenschr 2006;156:329-31.

http://dx.doi.org/10.1007/s10354-006-0303-8

14.Dutta D, Vanere P, Gupta T, Munshi A, Jalali R. Factors influencing activities of daily living using FIM-FAM scoring system before starting adjuvant treatment in patients with brain tumors: results from a prospective study. J Neurooncol 2009;94:103-10.

http://dx.doi.org/10.1007/s11060-009-9810-y

15.Kendall FP, Provance PG. Músculos provas e funçôes. 4a. ed. São Paulo: Ed Manole; 1995, p.200-2.
16.Riberto M, Miyazaki MH, Jucá SSH, Sakamoto H, Pinto PPN, Battistella LR. Validação da Versão Brasileira da Medida de Independência Funcional. Acta Fisiatr 2004;11:72-6.

17.Ciconelli RM, Ferraz MB, Santos W, Meiāo I, Quaresma MR. Tradução para língua portuguesa e validação do questionário genérico de avaliaçẫo de qualidade de vida SF-36 (Brasil SF-36). Rev Bras Reumatol 1999;39:143-50. 18.Gerber LH, Vargo M. Reabilitação para pacientes com diagnóstico de câncer. In: DeLisa JA, Gans BM. Tratado de medicina de reabilitação: princípios e práticas. São Paulo: Editora Manole; 2002, p.1382-3.

19.Abranches MHS, Vidotto MC, Vianna PCP, Gaiolla PD. Reabilitação em neurocirurgia. In: Braga FM, Melo PMP. Guias de medicina ambulatorial e hospitalar UNIFESP/ Escola Paulista de Medicina: neurocirurgia. São Paulo: Editora Manole, 2005, p.709-13.

20.Karakaya M, Kose N, Otman S, Ozgen TJ. Investigation and comparison of the effects of rehabilitation on balance and coordination problems in patients with posterior fossa and cerebellopontine angle tumours. Neurosurg Sci 2000;44:220-5.

21.Hill CI, Nixon CS, Ruehmeier JL, Wolf LM. Brain tumors. Phys Ther 2002;82:496-502.

22.Huang ME, Wartella J, Kreutzer J, Broaddus E, Lyckholm L. Funcitonal outcomes and quality of life in patients wtih brain tumors: a review of the literature. Brain Injury 2001;15:843-56.

http://dx.doi.org/10.1080/02699050010013653 